\title{
Violences de l'énonciation, énonciations de la violence dans Faire semblant c'est mentir de Dominique Goblet
}

\author{
François Poudevigne \\ Université Paris IV - Sorbonne
}

Faire semblant c'est mentir est une autobiographie aux contours flous, où l'auteure Dominique Goblet a tenté durant douze années de cerner la nature des relations qu'elle entretenait avec certains de ses proches, au premier rang desquels ses parents ${ }^{1}$ (chapitres 1 et 3 ) et son compagnon, Guy Marc Hinant ${ }^{2}$ (chapitres 2 et 4). Un premier constat s'impose, selon lequel l'ensemble de ces relations se caractérise par une forme plus ou moins marquée de violence, qu'il s'agisse d'une violence verbale, morale ou plus explicitement physique. L'auteure se représente ainsi, tout au long de l'œuvre, comme prise en étau au sein d'un réseau serré d'agressions, de conflits, qui déterminent son être au monde autant que son entreprise autobiographique, et où ne subsistent que de rares îlots d'apaisement (paradoxalement et comme une victoire, dans ses relations en tant que mère à sa propre fille).

La question qui nous intéressera ici sera précisément de savoir, dans une perspective d'analyse stylistique, comment cette violence trouve à s'incarner dans l'œuvre. Là où d'autres autobiographes ont adopté en ce sens une démarche introspective ${ }^{3}$, préférant aborder le récit de leurs expériences douloureuses par leur versant intime, Dominique Goblet opte, quant à elle, pour un schéma résolument extrospectif, fondé sur l'interaction communicationnelle. Cette distinction fondamentale se traduit notamment dans les œuvres par un usage divergent des récitatifs: d'un emploi hégémonique dans les travaux de Mussat et de Neaud, où ils actualisent le monologue introspectif que l'auteur surimpose, en voice over, au récit de son expérience, ils sont en revanche quasiment absents de l'œuvre de Goblet comme si l'auteure s'interdisait tout recours à une quelconque forme de commentaire ; de glose introspective qu'autoriserait l'usage du récitatif. C'est au contraire dans la parole extérieure, l'usage que l'on en fait, la perception que l'on en a, que se concentre toute la violence de l'œuvre; le langage devient un lieu d'affrontement, un enjeu de pouvoir à travers ce qu'il dit mais aussi, évidemment, à travers ce qu'il tait, ou ce qu'il empêche de dire. Cette tension se réalise alors dans l'œuvre par le biais de dispositifs énonciatifs complexes et variés, qui parce qu'ils sollicitent tous les plans de

\footnotetext{
${ }^{1}$ Auxquels s'ajoute la belle-mère, Cécile.

${ }^{2}$ Dont une mention paratextuelle nous apprend qu'il est également le co-auteur de l'œuvre :

«Les textes des chapitres 2 et 4 ont été co-écrits avec Guy Marc Hinant ».

${ }^{3}$ Voir par exemple les albums Sainte Famille et Carnation de Xavier Mussat, ou encore les quatre tomes du Journal de Fabrice Neaud.
} 
l'énonciation (l'énoncé lui-même, mais aussi l'énonciateur et ses postures, le schéma d'interlocution ou encore le contexte d'énonciation), jouent de toutes les ressources (notamment graphiques, iconiques et spatio-topiques) offertes par la bande dessinée, refusant de s'en tenir à sa seule dimension verbale, et à l'opposition canonique entre récitatifs et bulles de parole. Ainsi, l'enjeu de cette étude sera d'évaluer ce que le médium peut apporter à la représentation de la violence énonciative en tant que phénomène multimodal. Nos outils pour cela seront donc tant ceux de l'analyse du discours que de la théorie de la bande dessinée, dans une logique de complémentarité que nous espérons la plus stimulante possible.

La richesse d'une œuvre telle que Faire semblant c'est mentir ne nous a pas permis de traiter ici l'ensemble des situations énonciatives qu'elle met en scène. Nous avons donc opéré certains choix, pour nous concentrer finalement sur les seuls chapitres relatifs aux parents (chapitres 1 et 3). Ce choix se justifie par une évidente continuité thématique, mais également dans une logique que nous pourrions qualifier de «dramaturgique »; ces deux chapitres ont en effet des fonctionnements relativement similaires : ils se déroulent à huis clos dans deux espaces domestiques distincts, sur deux temporalités clairement circonscrites, et sollicitent un nombre restreint et invariant de personnages; ils disposent en ce sens d'un fonctionnement quasi théâtral, qui favorise leur appréhension en tant que « scènes ». Il s'agira donc de se demander comment, par le recours à certains dispositifs énonciatifs spécifiques, l'auteure parvient à représenter dans ces «scènes » la violence des rapports qui l'unissent à ses parents. Nous commencerons pour cela par nous intéresser aux diverses caractéristiques qui fondent la violence de l'énonciation parentale, puis nous analyserons les conséquences de cette violence sur le plan interlocutif, avant d'interroger la façon dont Dominique Goblet, réduite au silence en tant que personnage, retrouve la parole en tant qu'auteure - tissant pour cela son œuvre de subtils motifs dialogiques.

\section{Prendre la parole : usages énonciatifs violents}

Si l'on se réfère à la définition que propose Émile Benveniste de l'énonciation en tant que «mise en fonctionnement de la langue par un acte individuel d'utilisation » (Benveniste 80), l'on constate que c'est avant toute chose dans cet «acte de production des énoncés» (80) de la part des figures parentales que s'origine la violence de l'œuvre. L'enjeu sera ici de se demander ce qui dans cet usage de la langue produit précisément cette violence - et nous allons voir que cela dépend de différents critères non exclusivement verbaux que la bande dessinée, en tant que médium polysémiotique, exploite pleinement.

Le premier marqueur de cette violence énonciative est l'importance accordée à certains registres de langue : la parole parentale est une parole qui se caractérise par son oralité, sa familiarité. Cela est perceptible dans 
certaines tournures syntaxiques («Tout! J'ai fait pour vous! », «C'est quoi alors? »), dans l'emploi d'interjections («hein! », « hé »), d'onomatopées («han », «mmh »), d'adverbes familiers («ben ») - mais surtout dans l'usage régulier de jurons (« Nom de Dieu de nom de Dieu $\left.»^{5}\right)$, de gros mots («Elle n'a rien à foutre chez moi! ») ou d'injures (" péteuse », "sale gamine », « sale petite garce »). L'on s'aperçoit alors que mal parler revient rapidement à mal se parler : ces différents marqueurs lexicaux, en plus de participer d'une sorte de réalisme linguistique, fonctionnent comme autant de catalyseurs de la violence en langue, et identifient les locuteurs qui en usent comme des locuteurs violents. Jurons et injures constituent en ce sens des sortes de points d'appui à l'épanouissement d'une violence énonciative plus complexe et plus globale, dont ils assurent la scansion, la structure rythmique.

Au-delà de cette dimension verbale, la violence énonciative s'ancre également dans un ensemble de données paraverbales, sur lesquelles l'auteure insiste fortement par le biais de moyens graphiques variés. Il s'agit de donner à entendre la parole parentale dans sa profération («le terme paraverbal renvo[yant] aux aspects vocaux des productions verbales » [Detrie et al. 233]) : la voix, le timbre, l'intonation de celui qui parle - et ce que cette voix et ce ton peuvent avoir de violent, car « ce n'est pas seulement le choix des mots et leur insertion dans l'axe syntagmatique qui sont signifiants, mais aussi l'expression vocale à laquelle ils sont associés. » (233)

Cela se traduit dans l'œuvre notamment par un usage hyper-expressif de la ponctuation, qui excède largement les conventions typographiques en vigueur. Certaines phrases sont ainsi ponctuées par deux, trois voire quatre points d'exclamation ou d'interrogation, ces deux signes se combinant parfois en une manière de comble plus canonique de l'expressivité en bande dessinée «?! »; d'autres fois encore, ils vont jusqu'à déborder le cadre établi par les contours de la bulle ou de la case, signalant de ce fait la dimension proprement excessive du ton. Cette modalité d'expression, dans la mesure où elle est d'un emploi hégémonique, voire systématique, dans l'œuvre (et indépendamment de tout contenu propositionnel), signale une forme d'hystérie énonciative qui par effet de système devient violente pour le lecteur, qui se heurte sans cesse à la même prolifération de signes : ici, l'on crie à chaque page. D'autant que l'auteure joue également de la graisse et de la taille des caractères pour rendre compte de la violence de l'intensité vocale. Ainsi dans l'œuvre, cette taille et cette graisse augmentent comme augmente le volume sonore des énoncés - et ce jeu sur l'intensité du tracé parvient en

\footnotetext{
${ }^{4}$ Toutes les citations de cet article sont issues des chapitres 1 et 3. L'album n'étant pas paginé, il est difficile de les référencer plus précisément.

5 À noter que Goblet renforce plus loin dans le chapitre 3, la familiarité de ce juron blasphématoire en tentant de lexicaliser la prononciation de son père [ « Nom de d'jeu de nom de d'jeu »] - transformation qui en renforce, par effet de sonorisation, la densité et la violence.
} 
certains endroits à créer un effet de vacarme proprement dérangeant : on a le sentiment de ne plus s'entendre lire ${ }^{6}$.
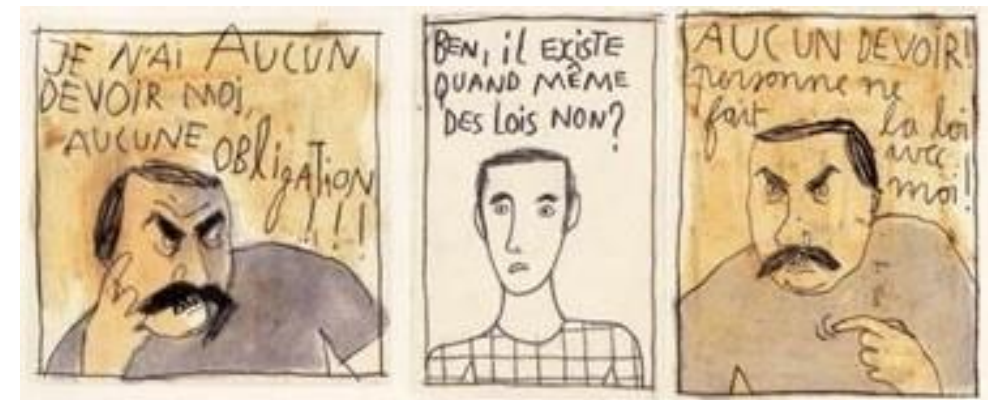

Image 1 : (Goblet 20) $@$ (2007, Dominique Goblet \& L'Association

L'intensité sonore des énoncés peut enfin se traduire par l'usage expressif des quelques couleurs que l'on rencontre dans l'œuvre. Dans ce strip par exemple (Voir Image 1), le rugissement du père se fait entendre par la superposition, sur l'image, d'une couche de couleur sépia qui "colore» littéralement son discours; la case est, à l'image du locuteur, comme échauffée : la violence réside alors dans la rupture chromatique qui s'établit d'une case à l'autre. Une fois encore, cette violence énonciative existe dans l'œuvre sous la forme graphique d'un surplus, d'un excès : l'auteure met en place toute une économie de la violence qui est une économie de l'ajout, du trop-plein, au cœur de laquelle tentent de subsister quelques «zones neutres », comme la case centrale du strip ici concerné.

Enfin, un certain nombre d'éléments strictement non verbaux participent également du processus énonciatif, et de la violence qui dans l'œuvre s'y déploie. On nommera cet ensemble, à la suite de Jacques Cosnier, « gestualité communicative »(Charadeau 285). Il peut s'agir en premier lieu des mimiques faciales, ou "gestes expressifs faciaux» (285), qu'adoptent les énonciateurs: Goblet accorde en ce sens une grande importance aux éléments mobiles du visage que sont les yeux, la bouche et les sourcils, qu'elle représente souvent furieux ou menaçants. Ainsi, chaque figure parentale est représentée à un moment de l'œuvre les sourcils froncés, la bouche pincée ou béante, le regard noir, dans une attitude qui participe complètement de la violence globale de l'énonciation. Ces éléments atteignent une expressivité caricaturale dans le cas de la belle-mère,

Cécile (Voir Image 2), dont le visage s'apparente davantage à une tête de mort qu'à une quelconque face humaine - et dont la violence intrinsèque est ainsi renforcée par la rupture, tout aussi violente, qu'elle établit avec le système figuratif valable par ailleurs.

${ }^{6}$ On consultera, à propos de la dimension sonore de l'œuvre de Dominique Goblet, l'excellent article que Catherine Mao consacre à ce sujet. 

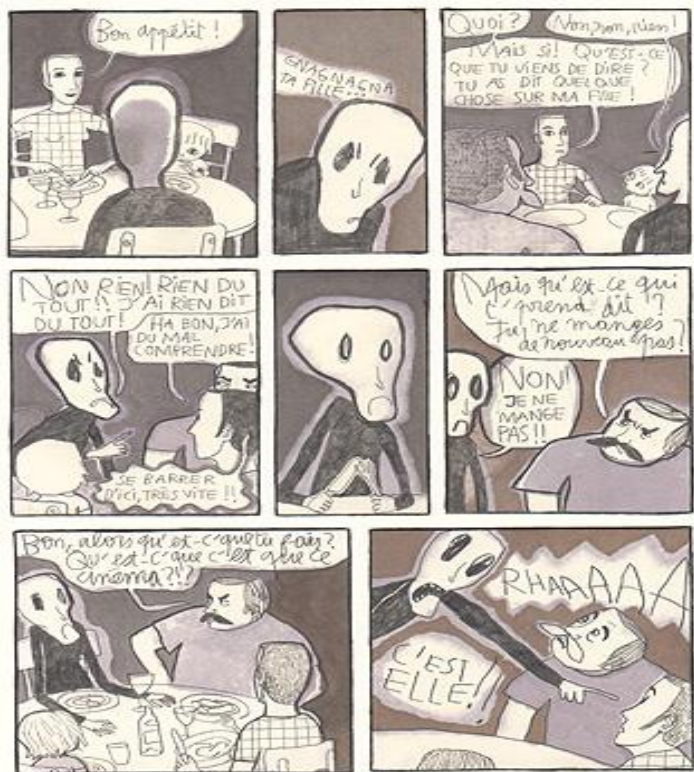

Image 2 : (Goblet 114) (C) 2007, Dominique Goblet \& L'Association

Le corps des énonciateurs peut également se montrer violemment éloquent, et à l'instar du visage, certaines zones sont en ce sens privilégiées, dont notamment les mains. Le père ou Cécile pointent ainsi un doigt accusateur sur Dom, renforçant par ce "geste déictique » (Charadeau 286) l'accusation qu'ils sont en train de formuler («Quoi?! »/ «Raaah! C'est elle! » [Voir supra Image 2, case 8]) - et ce doigt institue Dom en coupable de manière encore plus directe (et partant, plus violente) qu'un énoncé de type verbal. Si la violence du geste, en l'occurrence, reste symbolique, elle peut devenir en certaines autres occasions plus explicitement physique : c'est ainsi que l'on observe une gradation dans les réactions violentes de la mère au cours de l'épisode du grenier (chapitre 3): aux remontrances verbales, s'ajoutent rapidement d'éloquentes expressions faciales, qui débouchent inévitablement sur une première empoignade par le bras; l'enfant ne se calmant pas, la mère se met à l'insulter (« sale gamine! ») et à l'empoigner par les cheveux, préfigurant l'acmé du châtiment corporel final (Voir infra Image 8 b). Dans cette séquence, chaque empoignade s'accompagne d'une sévère remontrance: l'on voit bien ainsi comment au sein de l'énonciation maternelle le geste relaie la parole en une surenchère permanente d'actes violents.

\section{L'impossible dialogue}

Qu'il s'agisse de la dimension verbale, paraverbale ou non verbale de l'acte énonciatif, l'on voit donc bien comment l'énonciation parentale est activement violente dans ses conditions mêmes de production. Or nous 
allons voir qu'elle n'est pas seulement violente dans son affirmation brutale, mais également parce qu'elle implique la négation d'une parole autre - qui serait celle de Dom.

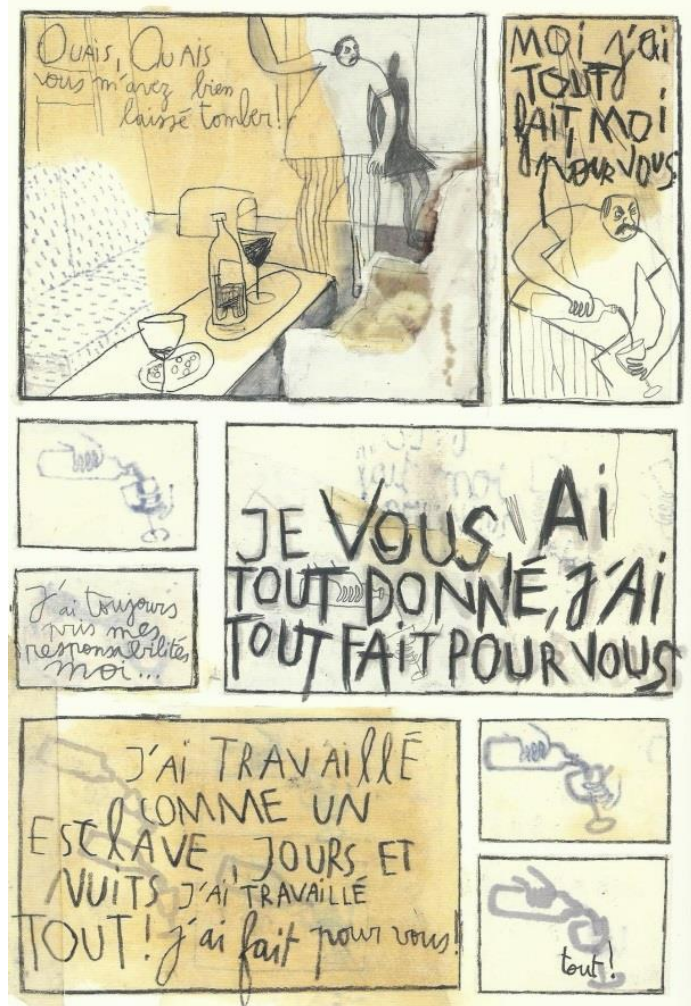

Image 3 : (Goblet 18) @ 2007, Dominique Goblet \& L'Association.

D'abord, car le discours du père (chapitre 1) est un discours sans extérieur, sans appel; il est régi par un effet de boucle, de reprise des mêmes formules qui fonctionnent dans l'album comme autant de leitmotivs. Il donne ainsi l'impression d'être replié sur lui-même, en une logique de ressassement permanent: c'est un discours qui se définit moins par une idée de progression thématique que par la reformulation perpétuelle des mêmes reproches. On pourrait ainsi dire qu'il s'agit d'un discours qui vit au risque du monologisme - c'est-à-dire qu'il n'est traversé, qu'il ne prend en compte (sciemment ou inconsciemment) aucun discours extérieur, pour se définir exclusivement vis-à-vis de lui-même: c'est un discours rigoureusement hermétique à la parole de l'autre, ce qui constitue, sur le plan énonciatif, une nouvelle forme de violence. Le père s'extrait ainsi de l'interaction verbale : il ne parle plus avec, ni même à, il se contente de parler, sans rien vouloir entendre.

Cette violence se traduit dans l'œuvre de deux façons différentes : par l'exploitation de la spatialité des inscriptions graphiques d'une part; par certains procédés de mise en page d'autre part. Si l'on prend l'exemple d'une 
planche comme celle-ci (Voir Image 3), l'on s'aperçoit d'abord que la parole du père est régie par cet effet de boucle que nous venons de décrire (il ne fait que reformuler de plusieurs manières la même idée culpabilisante : "J'ai tout fait pour vous »); or pour relayer visuellement cette insistance excessive, l'auteure vide progressivement les cases de tout contenu autre que cette sentence et ses reformulations - si bien que le discours obsessionnel du père finit par prendre littéralement toute la place (cases 5 et 6 ) : il n'est aucune figure, aucun discours qui puisse apparemment ${ }^{7}$ coexister avec lui. D'autant qu'en jouant ici aussi sur la taille et la graisse des caractères, Goblet rehausse encore ce que ce propos peut avoir d'écrasant. Elle ira même, deux pages plus loin (Voir infra Image 5), jusqu'à le faire figurer en un strip horizontal en tête de page, à la manière d'un titre qui pèserait sur la séquence suivante de tout son poids - et qui tiendrait éloignés, barrière infranchissable, Dom et son père au sein de la même case. D'autres procédés de mise en page relaient cette idée d'enfermement; dans un strip comme celui-ci (Voir Image 4), le père apparait comme pris au piège de son propre discours : la densité et la répartition spatiale des inscriptions verbales redoublent l'effet de clôture inhérent au cadre de la case, et l'étroitesse du découpage ${ }^{8}$ renvoie à l'étroitesse du raisonnement - au sein duquel il se tient seul, campé sur ses positions.
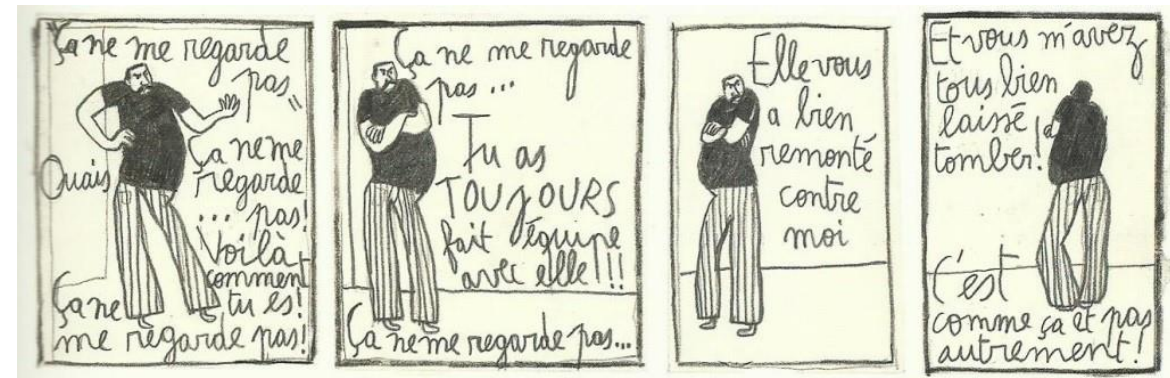

Image 4 : (Goblet 21) @ 2007, Dominique Goblet \& L'Association.

La conséquence directe, sur le plan interactionnel, d'un tel enfermement est que cela invalide d'emblée toute tentative de réponse de la part de son interlocutrice : le discours paternel, du fait de sa circularité, cherche à exclure Dom de la sphère dialogale - et rompt du même fait le contrat communicationnel qui suppose notamment une alternance régulière des tours de parole (dont Véronique Traverso nous dit qu'ils constituent «l'unité essentielle d'organisation des productions orales dialoguées. » [Charadeau 580]). À l'image de cette obsession paternelle, l'énonciation parentale est violente dans la mesure où elle cherche à s'imposer absolument, à s'extraire de

\footnotetext{
${ }^{7}$ Nous verrons comment le maintien discret d'un unique motif, celui de la bouteille, nuance subtilement ce constat.

${ }^{8}$ Il s'agit d'une des deux seules occurrences de l'album où Goblet use sur le plan horizontal d'un découpage en quatre cases, elle qui travaille sinon sur des formats de plus grande ampleur.
} 
toute forme d'interaction discursive - et partant, de réduire Dom au silence.

Cela passe d'abord par diverses stratégies d'invalidation de son discours, dont la plus récurrente est une forme dépréciative de reprise autonymique l'autonymie désignant «l'emploi d'un mot ou d'un syntagme en mention et non en usage : le locuteur, dans ce type d'emploi, construit un commentaire sur le fonctionnement linguistique du mot en question. » (Detrie et al. 44). En effet, il est fréquent que les interlocuteurs de Dom réagissent davantage à la manière qu'elle a de s'exprimer (à son dire) plutôt qu'au contenu propositionnel qu'elle exprime effectivement (son dit) : qu'il s'agisse de son père ou de Cécile, le flux conversationnel est fréquemment interrompu par la reprise et la répétition moqueuse de termes ou d'expressions employés par Dom : «Ça ne me regarde pas » (Voir supra Image 4), "Vieille France », «Là d'sus »... L'exemple le plus éloquent se rencontre dans cette planche (Voir Image 5), où le père réagit outrageusement à la locution «cela dit », qu'il répète six fois en l'espace de cinq cases. Cette répétition confine à la stigmatisation du fait de la mimo-gestuelle maniérée qui l'accompagne, de la typographie contournée qui traduit l'ironie du ton, et des formules agressives qui l'entourent ( $\mathrm{Tu}$ vas pas venir faire ta péteuse avec moi », «Toi et ton langage u-ni-ver-si-taire $\left.»{ }^{9}\right)$. Il y a dans ce procédé une triple forme d'anéantissement du propos de la jeune femme, puisque son interlocuteur d'abord lui coupe la parole, ensuite décide de se focaliser sur une dimension non pertinente de l'énoncé (refusant du même coup d'entendre ce qu'il pouvait contenir d'important au-delà des termes choisis), et le vide enfin littéralement, par le principe de répétition, de son contenu sémantique. Il est donc ici fait un usage destructeur de la reprise autonymique, qui cherche à empêcher Dom de se poser en énonciatrice crédible

${ }^{9}$ Le plus fort est que cette agressivité retourne la problématique de la violence au profit du père - qui se constitue en victime au sein de son propre discours, et justifie du même coup son recours à la violence en légitime défense. 


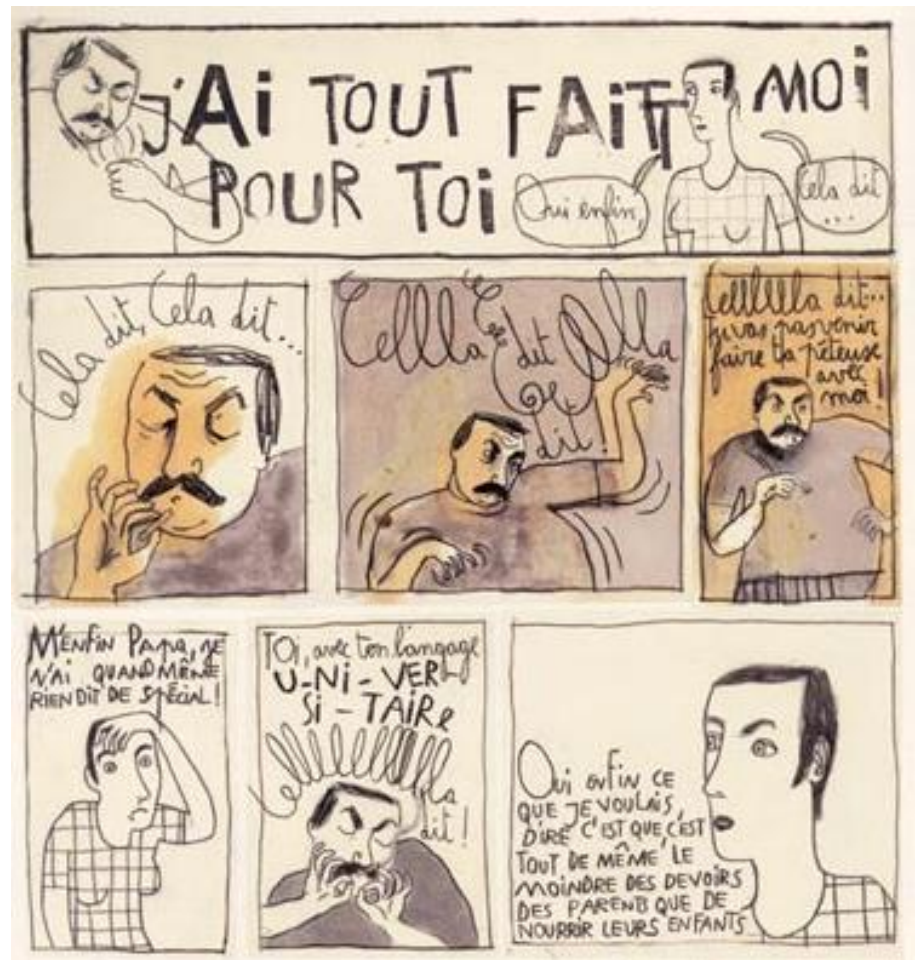

Image 5 : (Goblet 20) @ 2007, Dominique Goblet \& L'Association

D'autres procédés traduisent différemment les efforts fournis par les diverses figures parentales pour la réduire au silence: ainsi de cette transcription graphique du grondement paternel (Voir Image 6, cases 2 et 5), qui renvoie à une pratique que l'on pourrait dire coercitive de l'énonciation. Dom apparait comme ensevelie, opprimée sous la parole du père, dont la monumentalité graphique relaie la dimension négative (voire négationniste) de l'énoncé lui-même : une fois encore, la transcription visuelle du dire (casse, graisse, taille de la police, usage de la ponctuation) augmente la violence verbale du dit; la figure de Dom disparait progressivement sous une parole paternelle de plus en plus écrasante, de plus en plus envahissante, de moins en moins contenue (les bulles, encore présentes dans les cases précédentes, disparaissent progressivement). L'on notera que dans ces cases ne subsiste du père que sa parole : il n'est plus représenté physiquement, et sa présence est toute entière condensée dans son propos, en une sorte de concentration métonymique sur la seule manifestation de sa violence. 

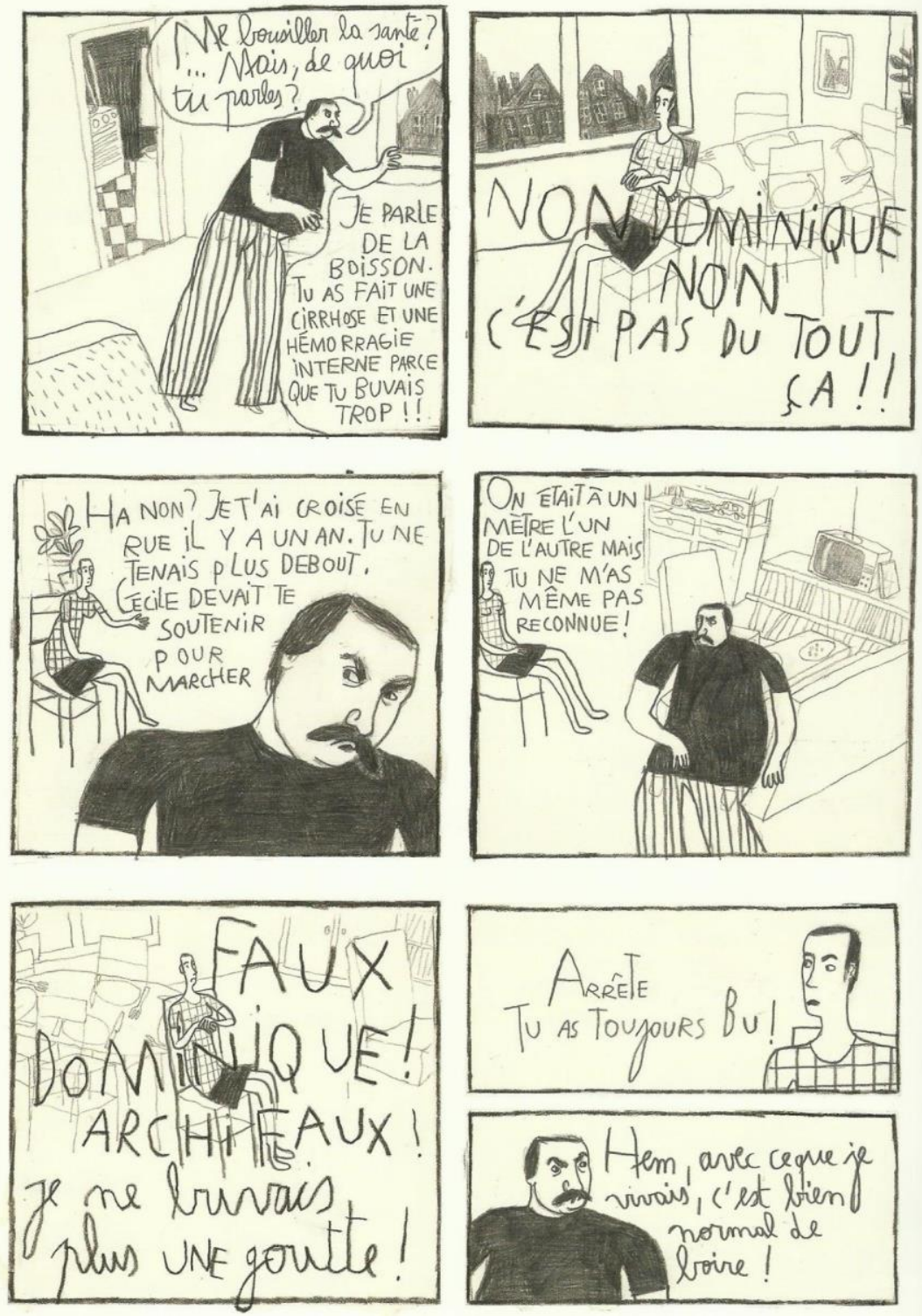

Image 6 : (Goblet 22) @ (2007, Dominique Goblet \& L'Association.

Enfin, dans la scène du grenier, cette injonction au silence atteint son paroxysme. Dans la mesure où le foyer perceptif de la scène se situe dans le salon (où se situe la télévision), ne nous parviennent de la dispute que les cris de la petite fille et de sa mère, les deux voix se distinguant, au-delà du propos, par l'usage des capitales pour la mère et des minuscules pour la fille (si bien que s'établit déjà, dans le lettrage même, une première forme de domination). Si ces deux voix coexistent un temps, l'injonction au silence adressée à Dom par sa mère interrompt par son extrême violence cette logique (« Ferme-la!! Tu vas la fermer oui?!! »), réduisant l'enfant au mutisme. En la privant de parole, sa mère la prive d'existence, dans la mesure où sa voix constituait jusqu'alors la dernière marque concrète, tangible de sa présence dans 
l'œuvre $^{10}$. En poussant à son comble cette logique de coercition énonciative, l'autorité parentale parvient donc à remettre en cause l'existence même du personnage de Dom. Plus que jamais, empêcher de dire revient à empêcher d'être - ce qui constitue, il faut l'admettre, une forme superlative de violence.

\section{Faire auvre : le mot de la fin?}

La violence énonciative est donc actualisée, dans l'œuvre, par l'usage que font les parents de leur propre discours, mais également par les conséquences que cela entraîne pour le discours de Dom; celle-ci apparait, à tous les âges, comme violemment remise en cause dans son statut d'énonciatrice, et comme sans cesse évincée, tenue à l'écart des structures communicationnelles qui s'élaborent à son entour. Mais si le personnage de Dom semble bien dans l'œuvre privée de parole ; ne peut-on voir dans le fait même de faire cuvre, pour l'auteure Dominique Goblet, un moyen de reconquérir cette parole étouffée?

La bande dessinée, dans son fonctionnement même, lui offre en effet la possibilité de développer un discours sous-jacent qui vient contrarier la violence énonciative parentale, et lui opposer une parole libre et contradictoire: on est alors confronté à divers procédés d'hétérogénéité énonciative, où l'auteure exprime, par un discours disjoint, un point de vue sur le discours parental qu'elle consigne. Cela se traduit d'abord par le déploiement d'une parole intérieure, qui en certains endroits établit comme un réseau contrapuntique répondant à la violence environnante.

La façon la plus évidente, en bande dessinée, d'exprimer ce type de parole est le recours à la bulle de pensée. Goblet y recourt à plusieurs reprises au cours de l'épisode paternel, chaque fois pour exprimer la réaction de son personnage vis-à-vis du discours ou de l'attitude de Cécile et du père. On se rend alors bien compte qu'il s'agit pour l'auteure d'une solution médiane permettant de ménager le statut raisonnable de son personnage (qui contrairement aux autres adultes ne se laisse pas ouvertement aller à des actes d'incivilité ou de violence) - tout en exprimant malgré tout une sorte de violence rentrée (en l'occurrence, lexicale : « Est-ce que j'ose la laisser partir avec cette givrée?? », "Non, mais je rêve, ils sont bourrés, ils sont morts bourrés »), preuve de sa distance critique à l'égard de la situation. Si elle ne répond pas, si elle ne s'insère pas dans la violence énonciative ambiante, c'est donc moins par soumission que par maîtrise de soi : elle montre ainsi que la violence, si elle surgit parfois, peut et doit être canalisée.

\footnotetext{
${ }^{10}$ Un personnage de bande dessinée, lorsqu'il est hors champ, n'existe plus (à la différence du cinéma où l'acteur continue d'exister même lorsque son personnage n'apparait plus dans le cadre).
} 
De façon plus discrète, elle use ponctuellement d'un système de notation périphérique, qui marque une forme de décrochage métaleptique par rapport au reste de l'œuvre: en effet, il permet de superposer au schéma communicationnel diégétique un système d'adresse plus ou moins explicite à l'encontre du lecteur. C'est le cas notamment du seul et unique récitatif de l'œuvre, dont nous en avons vu en introduction qu'il était d'un usage rare, et que l'on rencontre dans la première case de l'album : « Mon père ne boit plus. Plus une goutte parait-il. Je ne l'ai plus vu pendant quatre ans. Ma fille aura quatre ans en juillet... c'est-à-dire le mois prochain. » Ces quelques phrases, du fait de leur site d'inscription, de leur concision et de la rareté du dispositif énonciatif au sein duquel elles s'insèrent, acquièrent un statut spécifique dans l'œuvre; la charge d'implicite qu'elles contiennent (l'alcoolisme du père, le gel des relations, le scepticisme de la fille) résonne tout au long du chapitre et détermine notre lecture du récit qui va suivre. En effet, nous savons, grâce à cette précaution liminaire, à quoi nous en tenir - et nous endurons en même temps que le personnage les débordements du père. Une fois encore, l'énonciation auctoriale déjoue en partie la violence énonciative diégétique par la prise de recul qu'elle autorise.

Enfin, l'auteure se livre par l'usage de tropes visuels (métonymie et métaphore) à une véritable entreprise de remédiation de la violence énonciative - qu'elle dépasse en la mettant en scène. Qu'il s'agisse d'en augmenter ou d'en atténuer les effets, de la dénoncer ou d'en rire, il est toujours question ici de se réapproprier, de commenter par le geste artistique la violence subie; parler non plus face à la violence, mais parler sur la violence.

C'est le cas notamment dans cette séquence (Voir supra Image 3) que nous mentionnions précédemment; si nous avions noté l'évidement progressif des vignettes de tout contenu figuratif, nous n'avions rien dit des seuls motifs à se maintenir; apparaissent en effet, en filigrane sous les vociférations du père, les contours schématiques, mais néanmoins identifiables d'une bouteille de vin et d'un verre à pied, qu'une même main inlassablement conjoint. Ces éléments sont progressivement détachés du contexte d'énonciation (où ils ne jouent d'abord qu'un rôle anecdotique : dans les premières vignettes, c'est à peine si l'on y prête attention) pour s'imposer dans toute leur charge symbolique. Le personnage de Dom se tait, n'oppose aucune résistance à son père, mais laisse à l'auteure le soin de lui répondre - ce dont elle s'acquitte par le biais de cette insistance métonymique sur les causes de l'alcoolisme paternel. Ainsi, chaque occurrence du motif de la bouteille vient affaiblir, subtilement nuancer les prétentions sacrificielles du père, lorsqu'il dit avoir «tout fait», "travaillé comme un esclave, jours et nuits », «toujours pris [s] es responsabilités». Il y a dans l'usage de cette métonymie une sorte d'obstination sourde, une corruption silencieuse du discours paternel qui progressivement, à mesure que le verre s'emplit, inversement se vide de toute crédibilité - et ne reste alors de la violence que l'apparence d'une gesticulation un peu vaine.

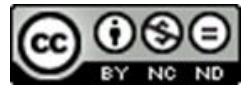


Dans la continuité de cette séquence se trouve l'une des planches les plus marquantes de l'album (Voir Image 7) où le père, sous les traits travestis d'une vierge à l'enfant, continue d'asséner ses éternels reproches. C'est en poussant à son comble la logique sacrificielle de l'éthos paternel (l'éthos étant cette « image de [lui-même] que le locuteur construit dans son discours pour exercer une influence sur son allocutaire » [Charadeau 238]) que l'auteure parvient à cette métaphore visuelle, dont l'inadéquation entre le comparant (la Vierge Marie) et le comparé (le père) révèle et dénonce l'impropriété du motif (la sainteté). En prenant le discours du père au pied de la lettre, en allant au bout de sa logique et en explicitant ce qu'il n'ose avouer, mais qui pourtant en constitue l'horizon outrancier, elle révèle sa dimension excessive (et partant, risible) en un geste paradoxalement iconoclaste ([« Il est évident que tu n'es pas ce que tu prétends être », paraitt-elle ainsi lui dire] et d'une concision extrêmement efficace. Elle applique en cela le précepte qu'elle confie à sa fille apeurée au chapitre $3:$ "Les choses qui font peur, il faut en rire ».

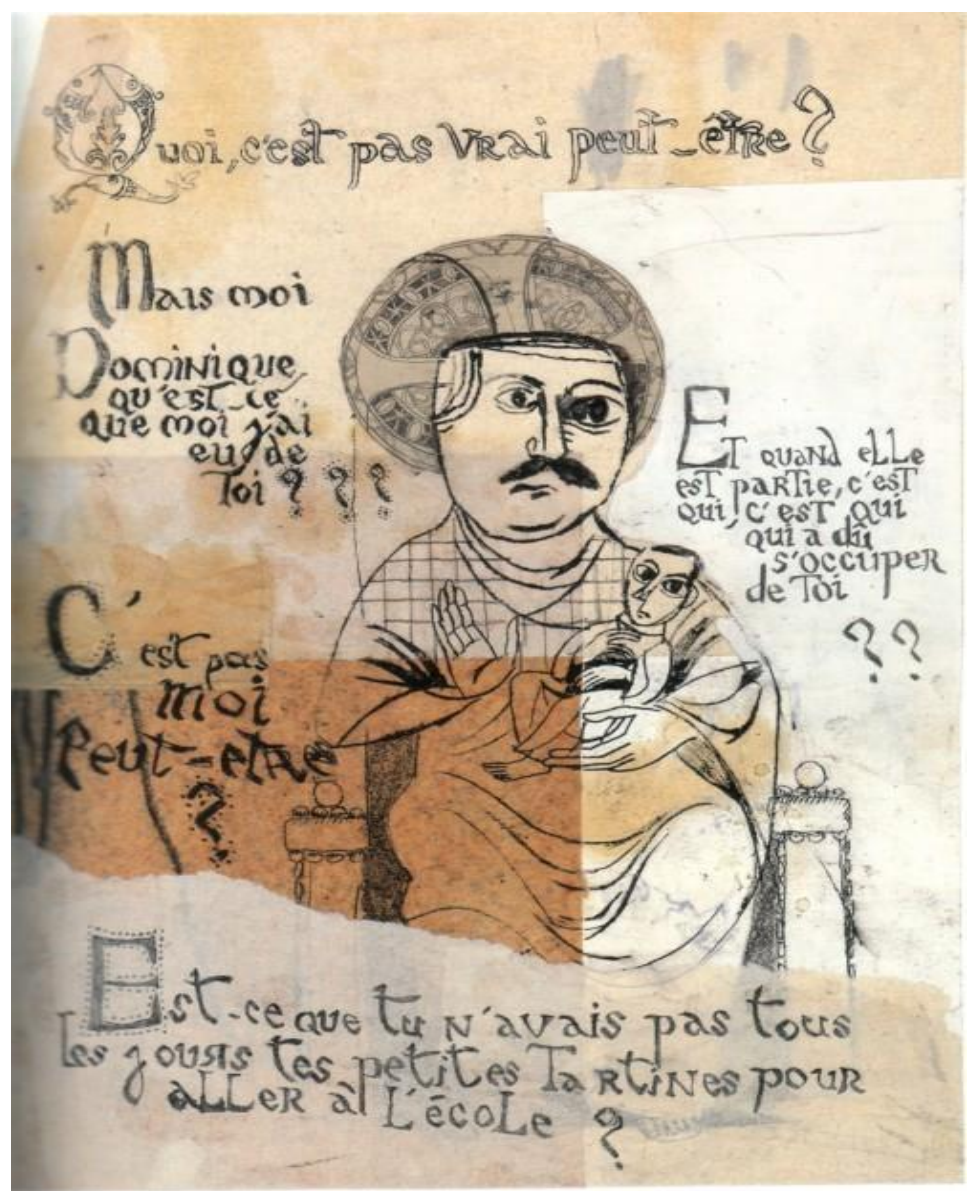

Image 7 : (Goblet 19) $\odot$ (2007, Dominique Goblet \& L'Association. 

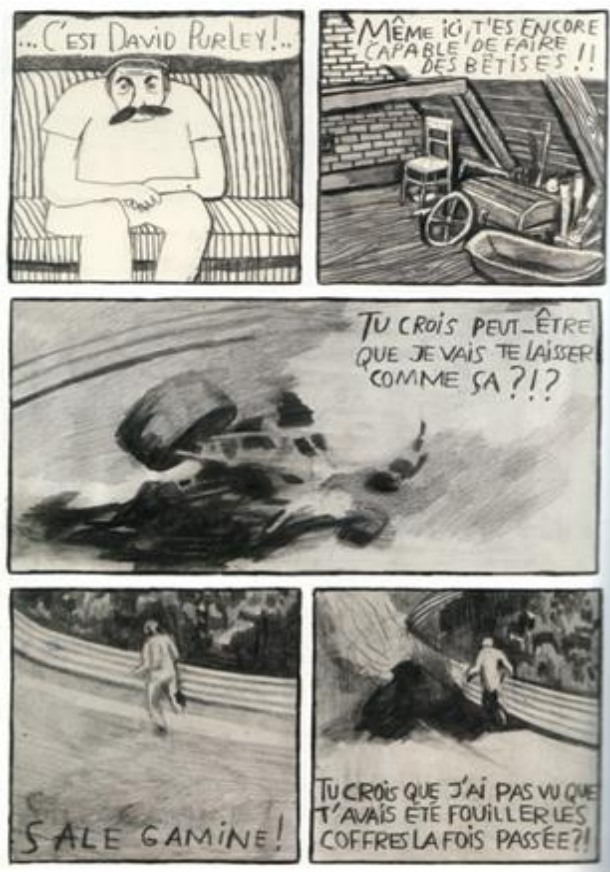

Image 8 a : (Goblet 104) @ 2007, Dominique Goblet \& L'Association.

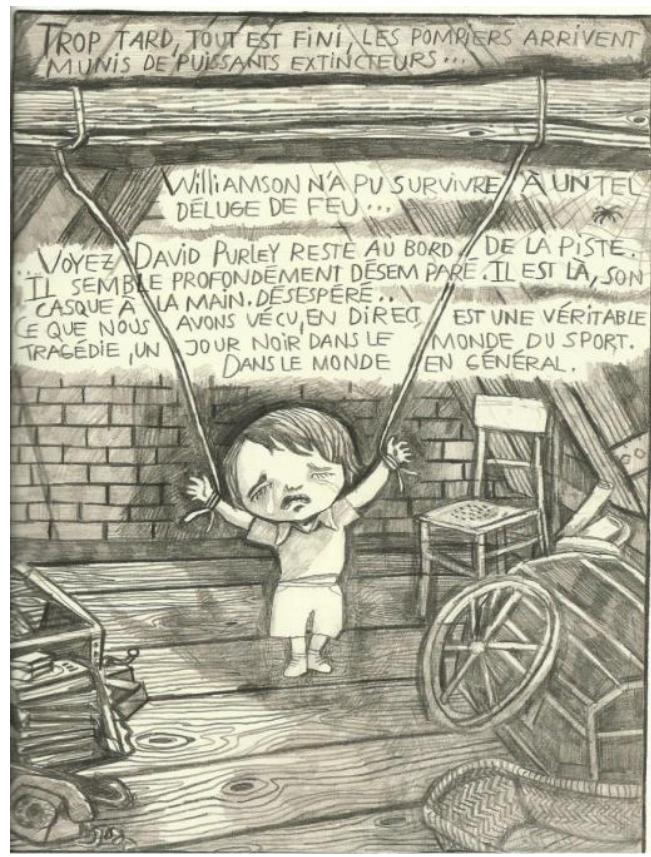

Image 8 b : (Goblet 107) (C) 2007, Dominique Goblet \& L’Association.

Enfin, au cours de la séquence du grenier qui constitue à tous points de vue une sorte de culmination de l'album (Voir Images $8 \mathrm{a} / 8 \mathrm{~b}$ ), l'auteure prend appui sur un accident survenu lors d'une course automobile pour essayer de dire, avec tant de force que de pudeur, la violence du châtiment 
imposé par la mère ${ }^{11}$. C'est ici l'exploitation du dispositif spatio-topique de l'album, «[...] c'est-à-dire [de] l'espace tel que la bande dessinée se l'approprie et l'aménage » (Groensteen, System 26-27), qui autorise un tel rapprochement, puisque le découpage et la mise en séquence tressent les images de l'accident avec le bruit de la dispute - et inversement, place l'image de la petite Dom en larmes sous le bruit des commentaires télévisés. L'auteure trouve alors, dans ce qui ne pourrait être qu'une simultanéité événementielle fortuite reconstruite a posteriori, le moyen d'établir une résonance [ou «similitude expérientielle globale» (Detrie 174)] de type métaphorique entre l'accident [devenant comparant] et le châtiment [comparé], permis par le motif de l'extrême violence. Pour dire la violence du châtiment, elle recourt à un discours exogène qui glose et illustre de façon oblique l'événement indicible; il s'établit alors tout au long de cette séquence un régime d'énonciation croisée, où textes et images se confrontent et se confondent en un déchaînement de violence qui confère à la scène toute son intensité. Il nait de cette confusion des différents plans de réalité à la fois une atténuation salutaire (le trope continuant d'être, malgré tout, une médiation) en même temps qu'un effet d'insistance à la limite du soutenable, du fait de la violence redoublée d'une part [le châtiment corporel se superposant à la mort d'un homme], et de l'attitude du père d'autre part; car la scène est bien perçue de son point de vue, lui qui ne parvient pas à se défaire de son poste de télévision alors même que lui parviennent les cris de sa fille - et c'est peutêtre en cela, dans cette focalisation qui dit la non-assistance que réside la violence ultime de la scène. Il n'en reste pas moins que la mise en œuvre (au sens propre]) de cette violence constitue pour l'auteure un moyen de se réapproprier enfin, par l'émergence du discours bédéique et l'implication d'un interlocuteur tiers (le lecteur, seul à même d'effectuer la synthèse des différents points de vue), l'expérience traumatique subie dans l'enfance.

Parvenu au terme de notre étude, si nous avons cerné les contours de la violence du discours parental et les manières dont cette violence était représentée, circonscrite, voire dépassée dans l'œuvre et le travail de l'œuvre, il resterait évidemment à effectuer le même travail pour les deux autres chapitres concernant la relation de Dom avec Guy-Marc, son compagnon.

\footnotetext{
${ }^{11}$ Au cours d'une après-midi pluvieuse, la petite Dom, désœuvrée, excède sa mère au point que celle-ci, pour la punir, décide de l'enfermer et de l'attacher dans le grenier de la maison. Le père, témoin distant de la scène, se lève d'abord pour empêcher la mère d'agir - avant de se rasseoir dans son canapé, happé par la retransmission télévisée d'une course automobile (circuit de Zandfoort, Pays-Bas, 1973), au cours de laquelle un pilote, Roger Williamson, trouve brutalement la mort. Dans un entretien accordé à Xavier Guilbert sur le site Du9, Dominique Goblet nous apprend toutefois que ce parallélisme fortuit est une invention personnelle, les deux événements n'ayant pas eu lieu simultanément (Groensteen, Un art 156). Nous y verrons ainsi la possibilité dramaturgique d'une mise en tension en même temps que d'une remédiation.
} 
Ces chapitres se distinguent de ceux que nous venons d'analyser dans leur production même, puisqu'ils ont été écrits à quatre mains par Dominique Goblet et Guy-Marc Hinant (qui nous offrent ainsi un exemple limite d'autobiographie plurielle), mais également dans la manière dont ils abordent cette question de la violence des rapports interpersonnels, et la formalisation énonciative de cette violence. À l'image du principe d'écriture, la violence, si elle est bien présente, est ici beaucoup plus diffuse, plus éclatée. Le personnage de Dom, non content d'être parfois privé de parole, va même jusqu'à s'absenter de certaines séquences où la violence, elle, continue d'être bien présente, mais sur un autre mode, moins frontal - comme au chapitre 2, lorsque Guy-Marc retrouve son ancienne compagne. La violence serait donc ici à appréhender suivant une dialectique du montré/dissimulé pleinement polyphonique.

Il resterait enfin à analyser la manière dont les dernières pages de l'album établissent une réserve dans l'œuvre, par le biais d'une dissolution progressive des divers éléments du code. C'est ainsi qu'en de grandes planches dépourvues de cases, de larges compositions abstraites accueillent les bribes d'une conversation téléphonique entre les deux amants, dont les voix progressivement s'amenuisent, se posent, s'apaisent. La violence alors se défait dans ces quelques planches d'où émane - enfin - une forme de sérénité, qu'il ne s'agit plus dès lors de vouloir montrer ni de vouloir dire, mais de confier plutôt au silence de l'image. 


\section{Bibliographie}

Benveniste, Émile. Problèmes de linguistique générale, 2. Paris : Gallimard, «Tel», 1974.

Charadeau, Patrick et Dominique Maingueneau, eds. Dictionnaire d'Analyse du discours. Paris : Seuil, 2002.

Détrie, Catherine. "Comme dit l'autre... L'autre, le corps et le réel dans le processus métaphorique ». Ed. Jacques Bres et al. Montpellier: Presses de l'université Paul-Valéry, 1998. 165-187.

Détrie, Catherine, Paul Siblot, et Bertrand Vérine, eds. Termes et concepts pour l'analyse du discours. Une approche praxématique. Paris : Honoré Champion, 2001.

Groensteen, Thierry. Système de la bande dessinée. Paris: PUF, «Formes sémiotiques », 1999.

Groensteen, Thierry. Un art en expansion. Dix chefs-d'auvre de la bande dessinée moderne. Bruxelles : Les Impressions nouvelles, «Réflexions faites», 2015.

Mao, Catherine. «L'œil et l'oreille dans Faire semblant c'est mentir de Dominique Goblet. D'un faire-semblant sonore à une esthétique sonore ». Images Re-vues. 1 Sept. 2009. Web. 25 décembre 2015. 